\title{
NUMERICAL METHODS FOR THE SIMULATION OF SEGMENTED CHIPS AND EXPERIMENTAL VALIDATION IN MACHINING OF TI-6AL-4V
}

\author{
C. Menze ${ }^{1 \star}$, R. Wegert ${ }^{1}$, T. Reeber ${ }^{1}$, F. Erhardt ${ }^{1}$, H.-C. Möhring ${ }^{1}$, J. Stegmann², S. Kabelac ${ }^{2}$ \\ ${ }^{1}$ University of Stuttgart, Institute for Machine Tools, Stuttgart, Germany \\ ${ }^{2}$ Leibniz University Hannover, Institute of Thermodynamics, Hannover, Germany \\ ${ }^{\star}$ Corresponding author; e-mail: christian.menze@ifw.uni-stuttgart.de
}

\begin{abstract}
The simulation of machining processes holds the opportunity for process improvement on many levels. Possible benefits that can be derived from accurate representations of the real processes on the tool from simulations include a prediction of tool wear, the shape of the chips produced, the forces, frictions and temperatures that arise and the residual stresses in the workpiece. These predictions can be used to improve the process in terms of its economic and ecological behaviour: Increasing the service life of the tools used through an improved understanding of the tool-workpiece interaction. The finite element method (FEM), among others, has emerged as a common method for simulating these processes.

When simulating machining processes using FEM, a major challenge is to avoid or compensate for the mesh distortions caused by the massive, fast-moving deformation processes, but at the same time to allow the mesh to be discretised in some way to ensure chip removal. To this end, various approaches will be presented in the course of this work and the mesh-based approaches will be explored in depth. Among other things, a remeshing approach for these investigations was developed. The machining of TI6AL4V is used to illustrate these approaches, as its tendency to form segmented chips is particularly challenging to model.
\end{abstract}

\section{Keywords:}

Machining, FEM, orthogonal cutting, element deletion, remeshing, ALE, CEL

\section{INTRODUCTION}

Machining processes have become an indispensable part of modern manufacturing. Their flexibility in shaping components is one of the main reasons for their widespread use in industries such as automotive and aerospace. Modern developments demand an improvement of the machining process in terms of productivity and ecology. To meet these requirements, it is essential to capture all the relevant process variables, such as the temperature or the prevailing stresses, to be able to make statements about the service life behaviour of the tool or the properties of the workpiece. However, the investigation of machining processes is difficult to implement experimentally due to the given conditions: high process speeds and difficult-toaccess process zones make it difficult to investigate even simple processes such as the orthogonal cut. The modelling of machining processes by means of discretisation methods such as the FEM offers the potential of an exact recording of all variables characterising the process.

In addition, most production processes, especially machining of high-strength materials such as titanium and titanium alloys, are usually carried out in combination with liquid cooling lubricants. This increases the degree of complexity considerably. The priority program "Efficient cooling, lubrication and transportation - coupled mechanical and fluid-dynamical simulation methods for efficient production processes (FLUSIMPRO)" (SPP 2231) funded by the German Research Foundation (DFG) is dedicated to the investigation and simulation of these complex thermomechanical and thermofluid interactions. In the first project period, the machining process and the flow situation are studied separately as submodels. Later, the submodels will be coupled with each other to investigate the interaction of cooling, lubrication, and transportation. The work presented here illustrates the investigation of the part model of simulation the chip formation.

The simulation of machining processes by discretising the continuum through finite elements is a well-researched area in general. However, the simulation of chip removal still poses problems. Extremely distorted elements and element deletions contribute to unstable simulations and noisy analysis results.

The increasingly emerging mesh-free particle-based machining simulation methods hold great potential to neutralise the chip removal problem [Heisel, 2013; Rana, 2019]. However, to make a qualitative statement about the resulting parts, they require a large quantity of particles, 
which are computationally expensive. A consideration of mesh-based approaches is still useful because they provide good information about the evolving geometries with less effort.

To use mesh-based simulation models, the problem of chip lift must be tackled. For this purpose, four mesh-based simulation models and their method of chip removal are compared. This is achieved by simulating a machining process involving TI-6AL-4V, which is considered a very hard-to-machine material, because of its special chip forming capabilities. To this end, a remeshing algorithm will also be developed.

In addition, the feed- and cutting curves of the models are compared with experimentally determined curves, to check the validity of the models. The FEM software used in this work is ABAQUS 2020.

\section{ANALYSIS OF MACHINING PROCESSES USING MESH-BASED FEM}

First, mesh-based simulation models will be briefly introduced. The focus of this introduction lays in its mechanism of chip removal.

\subsection{Lagrangian models}

The traditional way of modelling a cutting process is a lagrange model, in which the mesh is connected to the underlying material. Due to the lagrangian formulation, the associated meshes are also deformed when the underlying material is deformed. This is a problem when localised strains occur, as they can severely distort the meshes. Distorted elements can lead to inaccurate solutions and loss of stability, and in extreme cases the elements can become inverted, leading to negative element volumes and a termination of the simulation.

To achieve chip removal, the lagrangian models delete elements in the wider area of the tool radius. Element deletion can be invoked through the usage of certain material properties, such a damage initiation criterion and a damage evolution. If the end of the damage evolution process has been reached, the element is deleted. To achieve premature deletion of the elements before the tool radius, a sacrificial layer is introduced whose material values are lower than in the rest of the workpiece regarding the damage regime. A general approach of modelling a 2Dcutting process with element deletion can be seen in Fig. 1. The inclined area of the workpiece is intended to support the element distortions caused by the strain localisation of the shear plane for as long as possible by providing a mesh with an angle approximately equal to the shear plane angle. The explicit solver is suited to solve these models. The lagrangian model has been used successfully to model adiabatic shear banding [Öpöz, 2016; Jomaa, 2017].

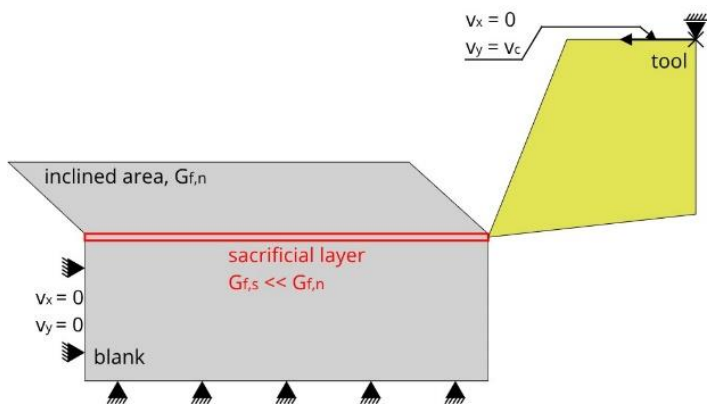

Fig. 1: Traditional Lagrange-model with element deletion

\subsection{Remeshing}

Remeshing is a technique used frequently in the simulation of processes where high gradients of strains occur (e.g., metal forming or cutting processes). There are several commercial software packages which offer this function (e.g. SFTC DEFORM, AdvantEdge).

A Lagrangian mesh is also used when remeshing. However, the solution of the problem is only advanced until distorted elements cause instabilities in the solution behaviour (convergence problems). The simulation is interrupted, and the contours of the workpiece are remeshed. This allows the mesh distortions to be resolved. A solution transfer of the field variables from the old to the new mesh takes place, called solution mapping. Remeshing criterions can be a specified error of the solution or the geometric properties of the elements (angles and characteristic element lengths).

Using remeshing, the simulation of a machining process behaves not like a removal of a chip with the creation of a new surface, but rather more like a deformation process with a flow of the metal around the tip of the tool [Bäker, 2004]. In that way, machining is considered like a metalforming process. Remeshing was successfully used to model segmented chip formation [Schulze, 2011; Umbrello, 2008].

\subsection{CEL models}

The coupled eulerian lagrangian-model is a very recent way of modelling machining. The special feature of the model lies in the fact that the workpiece is discretised in a Euler space whereas the tool is modelled as a lagrangian body. Originally reserved for modelling fluids, Euler spaces can handle the large deformations of the underlying material. Here, the Euler mesh is stationary while the underlying material moves through the mesh. From the point of view of the mesh, however, this is a lagrangian step, coupled with a remapping of the variables, especially in ABAQUS: a Eulerian time step is divided into a Lagrangian phase and a Euler phase. During the Lagrange phase, the nodes of the network are temporarily connected to the material. Elements therefore deform again with the material. This is followed by the Euler phase, in which the deformations are stopped. By exceeding a tolerance value, it is determined which elements have been distorted too much. These are re-meshed. The last step of the Euler phase is to determine the material flow between the elements, where material can move from one element to the other. In this material flow, the field quantities are also transferred between the elements by integrating the values from the old mesh over the new one. Non-distorted elements are excluded from the Euler phase to save computational costs, which is always a concern when using eulerian meshes. The totality of these steps thus leads to a stationary mesh. The initial conditions of the eulerian elements are specified through the eulerian volume fraction (EVF), which denotes the amount of material volume inside an element. EVF values between 0 and 1 denote a free surface. In eulerian elements, it is possible to have multiple materials within a single element. Technically, the empty space in eulerian elements is a material as well.

Through the usage of Eulerian elements, the otherwise occurring mesh distortions can be circumvented. Within the model, the tool is still modelled as a lagrangian body, hence the denotation.

The CEL-Model is especially suitable to model stationary cutting processes since an endless stream of blank material can be achieved through the application of suitable 
boundary conditions on the eulerian region. Therefore, the tool remains stationary and the blank moves towards the tool.

CEL-Models are known to handle segmented chip formation [Klocke, 2017; Benson, 2004].

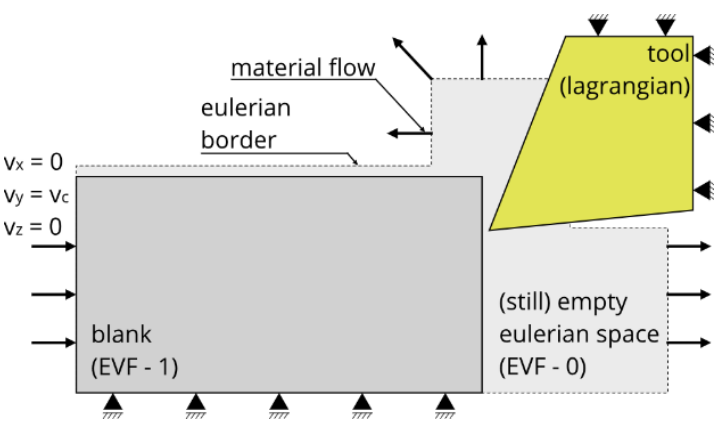

Fig. 2: CEL-model

\subsection{ALE models}

Arbitrary lagrange eulerian - elements combine the advantages of eulerian and lagrangian elements. This formulation takes advantage of both views in that the motion of the material is generally independent of the mesh on top of it, but the mesh can also be moved and deformed. Both move uniformly relative to each other but can better resolve mesh distortions due to the detached motion of the mesh [Soliman, 2020; Sridhar, 2020]. A smoothing algorithm improves the quality of the mesh topology by smoothing the nodes in a process called advection. In contrast to eulerian elements, the elements are always filled with one material. Since it is coupled with the mesh, not so severe deformations can be handled, but ALE in turn can give a precise outline of the evolving geometry.

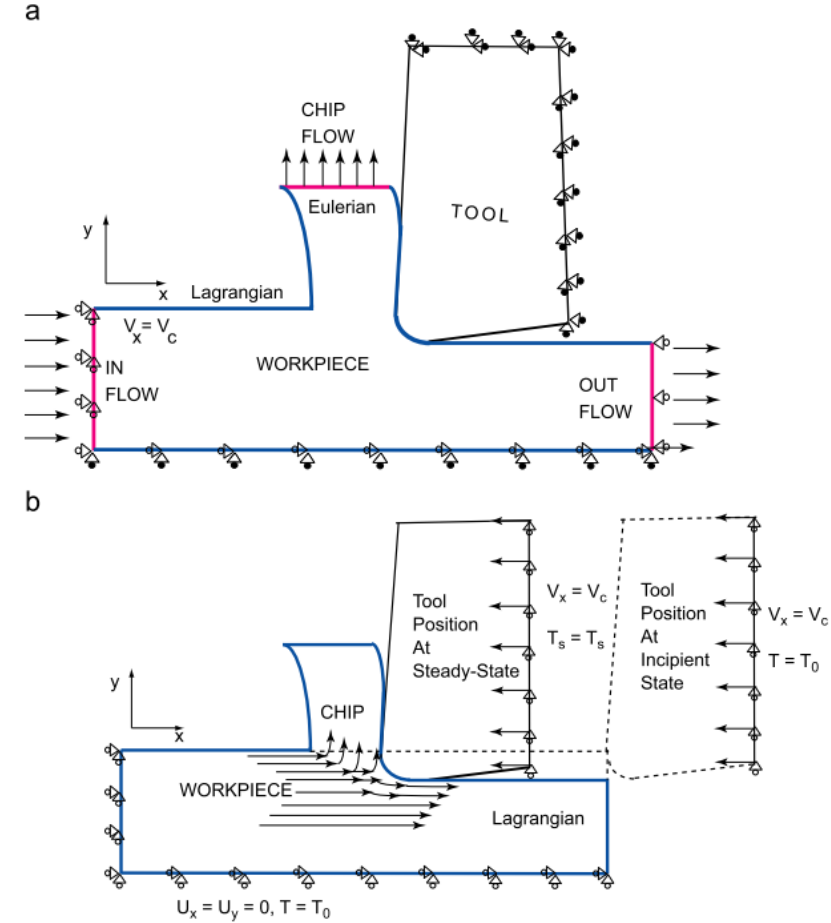

Fig. 3: Ways of modelling machining using the ALEapproach [Arrazola, 2010]

There are two ways of modelling machining with the ALEapproach, as shown in Fig. 3. One model assumes eulerian boundaries (a), which facilitate the in- and outflow of workpiece-/chip-material with an already pre-defined chip.
The second model assumes lagrangian boundaries and can form the chip from an incipient state (b).

Abaqus provides several smoothing algorithms to weigh either the distorted element volumes, the connected nodes or by using a higher-order weighing function. Zhang and Choi were able to simulate adiabatic shear banding using various ALE-formalisms [Zhang, 2021].

\section{MODEL SETUP}

The setup of all cutting models will be explained briefly. We restrict ourselves to the usage of 2D-Models according to the assumption of plain strain conditions. ABAQUS does not permit the usage of a 2D-Model when using eulerian elements. Through the restriction of all out-of-plane degree of freedoms we can achieve plain strain cutting conditions within the CEL-model.

\subsection{Material models and assumptions}

The Johnson \& Cook material model is widely used in machining simulations. Originally developed to describe material behaviour in impact tests, the Johnson \& Cook model is an empirical model that describes the yield behaviour of metals subjected to high strains, strain rates and temperatures [Johnson]. The model for describing the ideal plastic yield stress of a metal is:

$\sigma=\left[A+B \varepsilon^{n}\right]\left[1+C \ln \left(\frac{\dot{\varepsilon}}{\dot{\varepsilon}_{0}}\right)\right]\left[1-\left(\frac{T-T_{R}}{T_{m}-T_{R}}\right)^{m}\right]$

Where $A, B, n, C$, and $m$ are material-specific constants, $\varepsilon$ being the strain, $\dot{\varepsilon}$ the strain rate, $\dot{\varepsilon}_{0}$ the reference strain rate, $\mathrm{T}$ the temperature, $T_{m}$ the melting temperature and $T_{R}$ the reference temperature.

The first term denotes the strain induced hardening, the second term the strain rate induced hardening and the third term denotes the temperature induced softening.

Damage occurs by checking the incremental plastic strain increments towards a fracture-strain value:

$\omega=\sum \frac{\Delta \bar{\varepsilon}}{\bar{\varepsilon}_{f}}$

Where the fracture strain value is calculated using the Johnson \& Cook - damage model:

$\bar{\varepsilon}_{f, B}=\left[D_{1}+D_{2} e^{D_{3} \sigma^{*}}\right]\left[1+D_{4} \ln \left(\frac{\dot{\varepsilon}}{\dot{\varepsilon}_{0}}\right)\right]\left[1+D_{5}\left(\frac{T-T_{R}}{T_{m}-T_{R}}\right)\right]$

With $D_{1}-D_{5}$ being material-specific constants and $\sigma^{*}$ being the stress triaxiality.

When $\omega=1$, damage is initiated $(D=0)$. The softening regime now no longer follows a stress-strain relationship, because this would introduce mesh dependencies of the failure: When material softening occurs, plastic energy is dissipated. When the mesh is refined, less energy is dissipated as would be the case with a coarser mesh. To alleviate this problem, Abaqus uses the fracture energy of Hillerborg [Hillerborg, 1976]. Hillerborg denotes the required energy $G_{f}$ to open a crack of width $w$ in a material as:

$G_{f}=\int_{\bar{\varepsilon}_{f, B}}^{\bar{\varepsilon}_{f}} L \bar{\sigma}_{y} d \varepsilon=\int_{o}^{\bar{u}_{F}} \bar{\sigma} d \bar{u}$

With $L$ being the characteristic element length, $\bar{\varepsilon}_{f, B}$ is the plastic strain at the onset of damage, $\bar{\varepsilon}_{f}$ is the plastic strain at failure, $\bar{\sigma}_{y}$ is the yield stress, $\bar{u}_{F}$ is the completely plastic displacement at failure. A macron denotes the plastic regime of the variable. 
By dividing (4) through $L$, the equation can be traced back to a stress-strain relationship, as the fracture energy in respect to the characteristic element length resembles the area under the yield stress curve at the onset of damage, as depicted in Fig. 4. The softening of the material was defined by an exponential relationship:

$D=1-e^{\left(-\int_{0}^{\bar{u} \frac{\bar{\sigma}}{y} d \bar{u}} \frac{G_{f}}{G_{f}}\right)}$

With $D$ being the damage variable that denotes the loss of stiffness and the according reduction in yield stress. Upon reaching 1 (or rather 0.99 , since the exponent does not allow for reaching 1 completely) the element stiffness is completely degraded. If element deletion is flagged, the element will now be deleted and no longer partakes in the analysis.

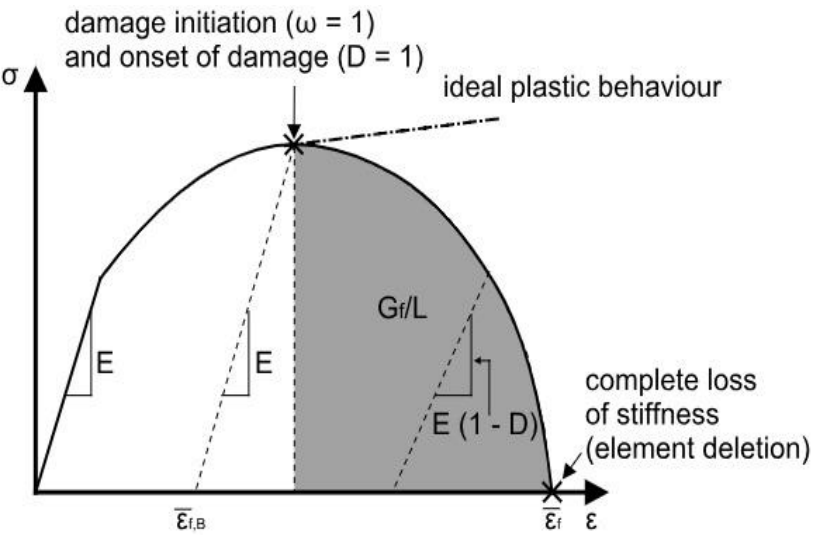

Fig. 4: Softening regime and fracture energy

When assuming plain strain condition as it can be reasonably be assumed when using the orthogonal cutting model, $G_{f}$ was found to be [Mabrouki, 2008]:

$G_{f}=K_{C, I, I I}^{2}\left(\frac{1-v^{2}}{E}\right)$ (plain strain)

With $K_{C, I, I I}$ being the fracture toughness for opening mode I or II. Within the framework of this work, only mode I was used. Plain strain conditions in orthogonal cutting can be assumed when the width of the cut $w_{p}$ exceeds the depth of the cut $a_{p}$ by a factor of at least 20 [Liu, 2017], although it should be noted that plain strain conditions are never strictly enforced in real experiments. But they are a reasonable approximation, since material flow perpendicular to the plane is small.

\subsection{Model setups}

The machining process is modelled as an orthogonal cutting process with a $2 \mathrm{~mm}$ by $0.5 \mathrm{~mm}$ blank. The tool is modelled with a dimension of $0.4 \mathrm{~mm}$ by $0.4 \mathrm{~mm}$, a clearance angle of $7^{\circ}$. The rake angle is varied between $10^{\circ}, 0^{\circ}$ and $10^{\circ}$. Contact is modelled with a constant coefficient of 0.15 and a thermal contact conductance of 10 $\mathrm{mW} /\left(\mathrm{mm}^{2} \cdot \mathrm{K}\right)$. The thermomechanical material parameters used in the simulations are depicted in

Tab. 1 and Tab. 2.

\section{Lagrange Model}

Lagrange models using a fracture energy-based element deletion were set up. It was found that models with negative rake angles as well as radii that exceed the thickness of the sacrificial layer are difficult to run and lead to difficulties when shear banding is initiated. Since the lagrangian mesh cannot form around a tool tip, the sacrificial layer must be extended to the height of the radius. When modelling adiabatic shear banding, this leads to a crack formation that precedes the progression of the tool, since the high temperatures along an adiabatic shear band invoke the damage evolution sooner than in the rest of the sacrificial layer. This results in a crack in the middle of the workpiece, and to severely distorted elements around the tool tip that will not be deleted.

We propose a different modelling approach. Rather than using an element deletion criterion based on a lower fracture energy within the sacrificial layer, a geometrical value based on the ratio of undeformed to deformed characteristic element length has been used. This can be achieved using Abaqus' *DELETE DISTORTED ELEMENTS function. In Fig. 5, the initial workpiece geometry and its partitions is shown. With this configuration, a stationary cutting state can be achieved with a tool radius and a negative rake angle.

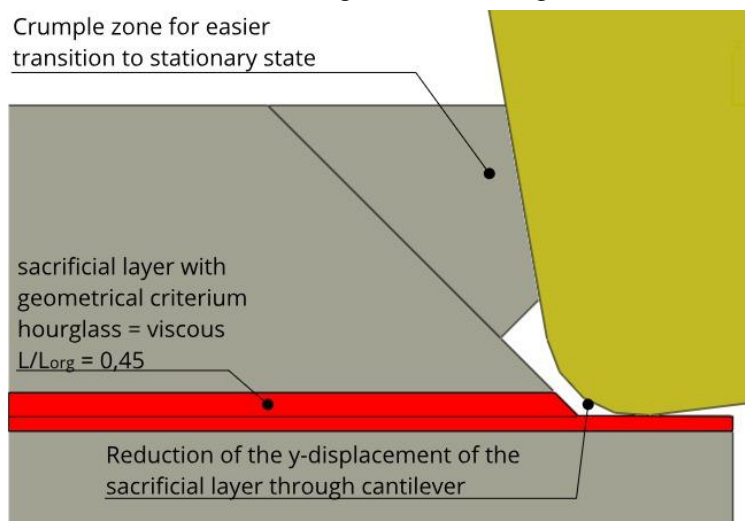

Fig. 5: Crumple zone, cantilever, and geometry-based sacrificial layer

Through the usage of this method, chip shear banding with negative rake angles and a tool radius can be modelled more easily. For a neutral rake angle, a comparison of both approaches can be seen in Fig. 4. The value of the element deformation ratio $\mathrm{L} / \mathrm{L}$ org $=0.45$ was determined by $a$ sensitivity analysis. A positive side effect of this model is the preservation of a relatively stable time increment because elements that are too heavily distorted are removed from the analysis.

While this approach does not eliminate the problem of element deletion per se, heat flux along the clearance angle and the new workpiece surface is now more pronounced since contact between radius and free surface of the workpiece can be achieved without losing simulation stability through severely distorted elements in the free surface.

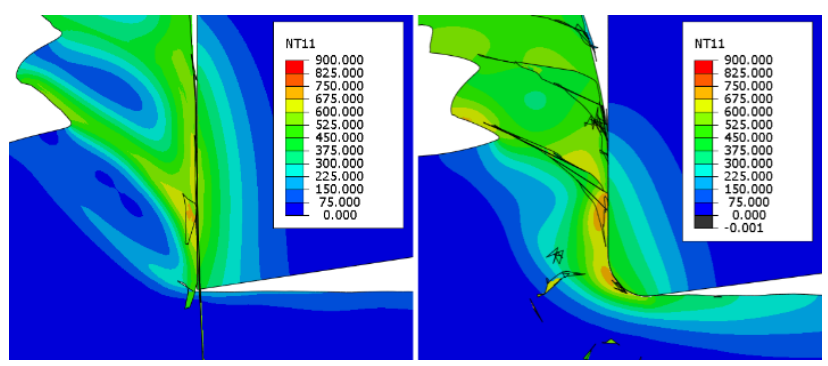

Fig. 6: Comparison between fracture energy-based sacrificial layer and element distortion based sacrificial layer

\section{Remeshing Model}

Abaqus offers a broad accessibility of all modelling functions via Python. Using this, a script was written, that manages the remeshing of the contours and the transfer of 
the solution using Abaqus' solution mapping function. The usage of the solution mapping function leads to a certain solution diffusion when mapping variables from the old mesh to the new mesh. A mesh close to the old mesh can alleviate this effect, hence remeshing is invoked rather frequent (time of the tool to travel through one of the smallest elements $(5 \mu \mathrm{m})$. As a solver, Abaqus only allows for the usage of the Standard-solver (Abaqus implicit solver) in combination with the *MAP function.

Within the framework of this solver, the Johnson \& Cook Damage initiation criterion as shown in (3) is not supported, unlike in the explicit models. Therefore, it was initially omitted. The model showed no sign of shear band formation, which implies that the temperature softening term of the constitutive model alone is not adequate to induce shear banding and that the strain softening of the damage evolution model is necessary to model segmented chips, as is confirmed by [Öpöz, 2016].

Therefore, a User routine in FORTRAN was written, that manages the point of damage initiation based on (3) and (2). When damage is initiated using this subroutine, damage evolution is defined using the fracture energy as described in (4). This ensures comparability as well as solution stability since the softening regime is critical regarding the convergence behaviour of the implicit solver, as it is considered a nonlinear material behaviour (alongside the plastic regime).
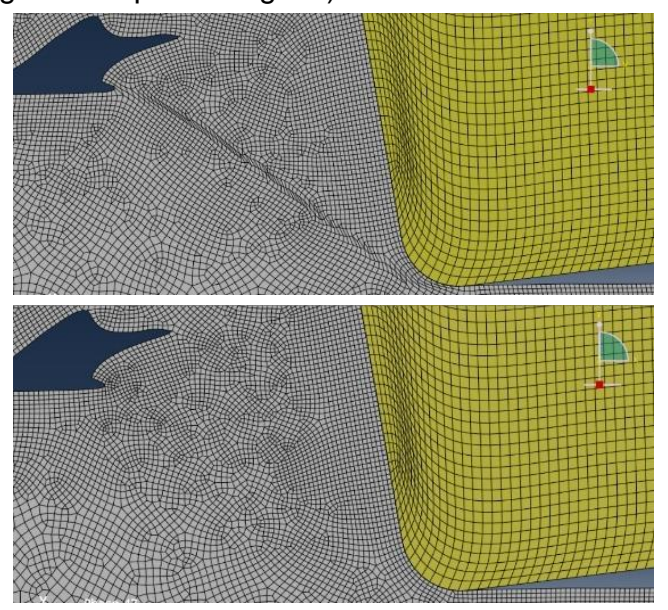

Fig. 7: Alleviation of mesh distortions along the shear band using remeshing

\section{CEL-Model}

A CEL-Model can only be modelled in a 3D environment. Through the usage of certain boundary conditions any movement perpendicular to the cutting plane was restricted. Like the other models, a fine mesh in the cutting region was used $(5 \mu \mathrm{m})$. The mesh coarsens with increasing distance to the cutting region, since eulerian elements are expensive. The region of the fine mesh is big enough to hold 1 - 2 segments. When the chip enters the coarse area of the mesh, the chip gets distorted, since the EVF is distributed over increased element volumes.
Euler material has the problem of sticking and adhering when it meets itself or with other Euler materials. When two Euler materials meet within a Euler element, they stick to each other because a single strain field is imposed on all materials within the element. To eliminate this problem, a one element high lagrangian distance holder is modelled, to avoid self-contact, as can be seen in Fig. 8 .

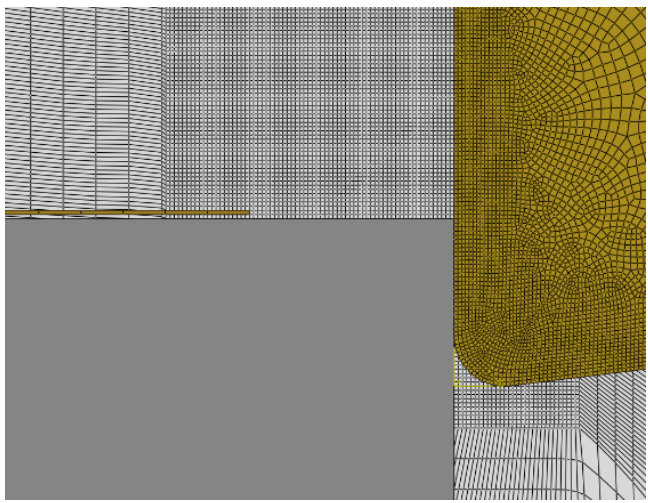

Fig. 8: CEL-Model und eulerian mesh domain

\section{ALE-model}

Since the goal was to determine the chip from an incipient state, model b from 2.4 was adapted. The first setup of the model did not include a damage model or element deletion. The usage of advection should facilitate chip removal by forming the blank material mesh around the tool tip, like in the remeshing model, see Fig. 9 left. The model failed to remove the chip. Further, as in the remeshing model, a damage evolution criterion is necessary to allow for shear band formation. The introduction of the model helped in forming an initial shear band, but the distortions exceeded the capabilities of the mesh advection when elements are deleted. A sacrificial layer proved to be a fatal, since the notches at the lagrangian-ALE-boundary region induced distortions in the ALE-region, which could not be handled by the smoothing algorithm, even when using frequent advection processes, see Fig. 9 right. The usage of neutral or negative rake angles proved to be especially problematic.

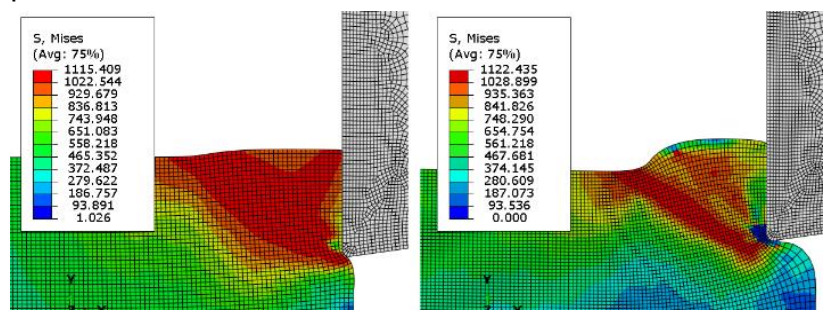

Fig. 9: ALE-model: no damage evolution (left) and damage evolution and element deletion: Damage evolution is necessary to facilitate shear banding. Nonetheless, it fails with the ALE-formulation (right picture is the last frame before the simulation fails).

Tab. 1: Mechanical parameters and J.\&C. constitutive model of Ti6Al4V used in the simulations [Ducobu, 2017]

\begin{tabular}{ccccccccccc}
\hline $\begin{array}{c}\text { young's } \\
\text { modulus } \\
{[\mathrm{GPa}]}\end{array}$ & $\begin{array}{c}\text { density } \\
{\left[\mathbf{g} \cdot \mathbf{c m}^{3}\right]}\end{array}$ & $\begin{array}{c}\text { poisson's } \\
\text { ratio } \\
{[-]}\end{array}$ & $\begin{array}{c}\mathbf{A} \\
{[\mathrm{MPa}]}\end{array}$ & $\begin{array}{c}\mathbf{B} \\
{[\mathrm{MPa}]}\end{array}$ & $\begin{array}{c}\mathbf{n} \\
{[-]}\end{array}$ & $\begin{array}{c}\mathbf{C} \\
{[-]}\end{array}$ & $\begin{array}{c}\mathbf{m} \\
{[-]}\end{array}$ & $\begin{array}{c}\dot{\boldsymbol{\varepsilon}}_{\mathbf{0}} \\
{\left[\mathbf{s}^{-1}\right]}\end{array}$ & $\begin{array}{c}\mathbf{T}_{\mathbf{m}} \\
{\left[{ }^{\circ} \mathbf{C}\right]}\end{array}$ & $\begin{array}{c}\mathbf{T}_{\mathbf{r}} \\
{\left[{ }^{\circ} \mathbf{C}\right]}\end{array}$ \\
\hline 115 & 4.43 & 0.22 & 870 & 990 & 0.25 & 0.011 & 1 & 1 & 1660 & 25 \\
\hline
\end{tabular}


Tab. 2: Thermal parameters, J.\&C. damage model and damage evolution parameters [Cervay, 1974]

\begin{tabular}{|c|c|c|c|c|c|c|c|c|c|c|}
\hline $\begin{array}{l}\text { Thermal } \\
\text { conductivity } \\
{\left[\mathrm{W} \cdot \mathbf{m}^{-1} \cdot \mathrm{K}^{-1}\right]}\end{array}$ & $\begin{array}{c}\begin{array}{c}\text { Specific } \\
\text { heat } \\
\text { capacity } \\
{\left[\mathrm{J} \cdot \mathrm{kg}^{-1} \cdot \mathrm{K}^{-}\right.} \\
1]\end{array}\end{array}$ & $\begin{array}{c}\text { thermal } \\
\text { expansion } \\
\text { coefficient } \\
{\left[\mathrm{K}^{-1}\right]}\end{array}$ & $\begin{array}{c}\text { Taylor- } \\
\text { quinney- } \\
\text { factor } \\
{[-]}\end{array}$ & $\begin{array}{l}D_{1} \\
{[-]}\end{array}$ & $\begin{array}{l}D_{2} \\
{[-]}\end{array}$ & $\begin{array}{l}\mathrm{D}_{3} \\
{[-]}\end{array}$ & $\begin{array}{l}\mathrm{D}_{4} \\
{[-]}\end{array}$ & $\begin{array}{l}D_{5} \\
{[-]}\end{array}$ & $\begin{array}{c}\mathrm{K}_{\mathrm{I}, \mathrm{C}} \\
{[\mathrm{MPa} .} \\
\left.\mathbf{m}^{-1 / 2}\right]\end{array}$ & $\begin{array}{c}\mathrm{G}_{\mathrm{f}} \\
{[\mathrm{N} /} \\
\mathrm{mm}]\end{array}$ \\
\hline 7.2 & 505 & 8.8 & 0.9 & -0.09 & 0.25 & -0.5 & 0.014 & 3.87 & 52.74 & 21.86 \\
\hline
\end{tabular}

\section{EXPERIMENTAL SETUP}

The orthogonal cutting experiments were conducted on a self-developed test stand at the IfW. The system consists of a single comb linear direct drive installed on a concrete machine bed with a guaranteed maximum speed of 180 $\mathrm{m} / \mathrm{min}$. Cutting and feed forces are detected on the workpiece side by a piezoelectric dynamometer. The clamping device for the workpiece is connected to the dynamometer. A high-speed camera is set up perpendicular to the direction of travel of the LDA to gain an insight into the chip forming behaviour. Fig. 10 shows a recording of the cutting process with segmented chip formation.

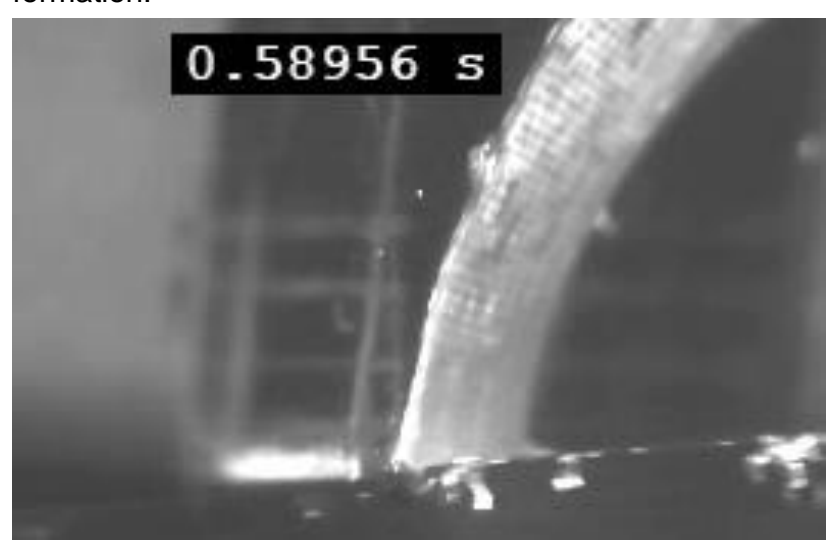

Fig. 10: High speed camera recording of shear banding

Chips were investigated by fixating them within a plastic casting with subsequent longitudinal grinding.
The experimental setup was designed around the pèclet number [Möhring, 2018]:

$P_{e}=\frac{v_{c} * a_{p}}{\omega}$

The pèclet number quantifies the heat energy dissipated from the tool tip per unit time by the sliding chip. Adiabatic shear band formation is promoted by a local heat build-up which is enabled by a lack of thermal conductivity. By keeping the pèclet number constant, a constant thermodynamic state can be maintained throughout the experimental design, which should facilitate the comparability of the experiments regarding shear band formation.

\section{RESULTS}

In this chapter the results of the experiments will be presented alongside the simulated results. The focus of this investigation lays in the comparison of the simulated and experimental forces. Chip morphology will also be discussed briefly.

\subsection{Feed- and cutting forces}

Fig. 11 shows the results of the simulated feed-and cutting forces as a percentage of the experimental forces. It can be noted that the improved element deletion model as well as the remeshing script achieve good results in the cutting force regime (deviations between 5\% and 25\%). In the element deletion model, a positive rake angle as well as high cutting speeds and small cutting depths lead to a dramatic drop in calculated forces.

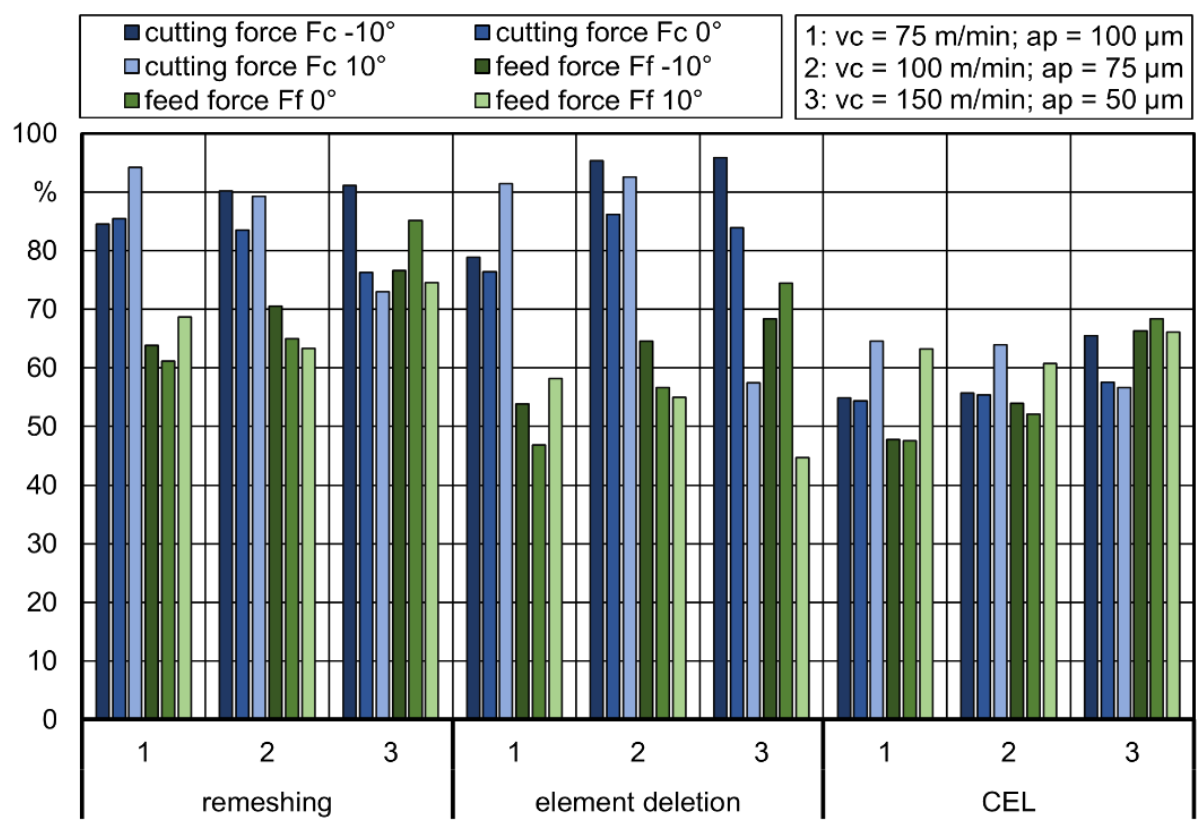

Fig. 11: Simulated cutting- and feed forces: percentage of experimental values MM Science Journal | 2021 | NOVEMBER - Special Issue on HSM2021 
Tab. 3: Different chip dimensions and segm. frequency (in percent to experiments over all cutting parameters)

\begin{tabular}{cccc}
\hline & Remeshing & Element deletion & CEL \\
\hline h1 & $243 \%$ & $236.8 \%$ & $103.95 \%$ \\
h2 & $130.26 \%$ & $143.66 \%$ & $85.37 \%$ \\
aa & $40.91 \%$ & $90.1 \%$ & $46.87 \%$ \\
bb & $50.64 \%$ & $107.26 \%$ & $58.47 \%$ \\
Ф & $121.5 \%$ & $108.42 \%$ & $120 \%$ \\
f & $131.37 \%$ & $64 \%$ & $110.34 \%$ \\
\hline
\end{tabular}

The feed forces could best be anticipated by the remeshing script, followed close by the element deletion model $(15 \%$

and $29 \%$ for the remeshing script, $26 \%$ and $55 \%$ for the element deletion model). But the deviation of the predicted forces to the real forces is more pronounced here. The loss of accuracy of the element deletion model in comparison to the remeshing model could be attributed to the element deletion itself, since frictional effects along the flank face are diminished, since a loss of contact could not be avoided The CEL-model underestimates cutting forces as well as feed forces by a margin of roughly $50 \%$, which is substantial. The error difference between feed and cutting forces is however smaller than in the other two models.

Generally, all models underestimate both feed- and cutting forces. This indicates inadequate material model parameters, especially of the constitutive model. The even lower feed forces can additionally be attributed to a low and constant friction coefficient. A more dynamically evolving friction coefficient should be adapted in future works.

\subsection{Chip morphology}

Experiments have been conducted. In Fig. 12, a segmented chip from the experiment is shown. Several dimensions of the chip have been examined: minimal and maximal segment height $h 1$ and h2, minimal and maximal segment width aa and bb, shear angle $\varphi$ and segmentation frequency f. Segment Fig. 14 shows a more direct comparison of the different evolving chips from the simulations and the experiment. It can be noted that the grade of segmentation can only be matched by the CELmodel, which is also shown by the values in Tab. 3. The element deletion model deletes the elements within the shear band, which causes the individual segments to penetrate each other, see Fig. 14 upper right. The remeshing model cannot match the segmentation shown by the experiment and tends to segment not as heavily as the other models. On the other hand, element deletion in the shear band in the element deletion models induces a notch effect, which further weakens the shear band. This leads to a more easily facilitated segmentation.

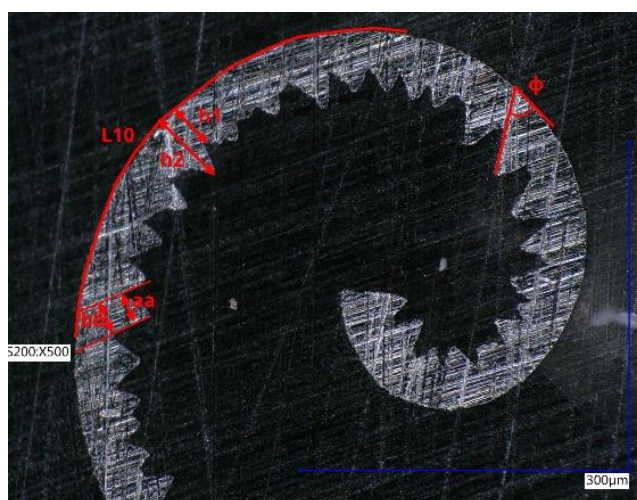

Fig. 12: Segmented chip from experiments (x500)

\subsection{Significance of the softening parameters}

Since the influence of the damage evolution parameter plays an important role in the formation of segmented chips, simulations with a variation of these parameters were conducted, as is shown in Fig. 13. It was found that a decrease in fracture energy leads to fractured chips in the element deletion model as well as in the CEL-model. In the CEL-model, material with no remaining stiffness moves around in the model, see Fig. 13 down left. In the remeshing model, the decrease in fracture energy led to a more pronounced segmentation.
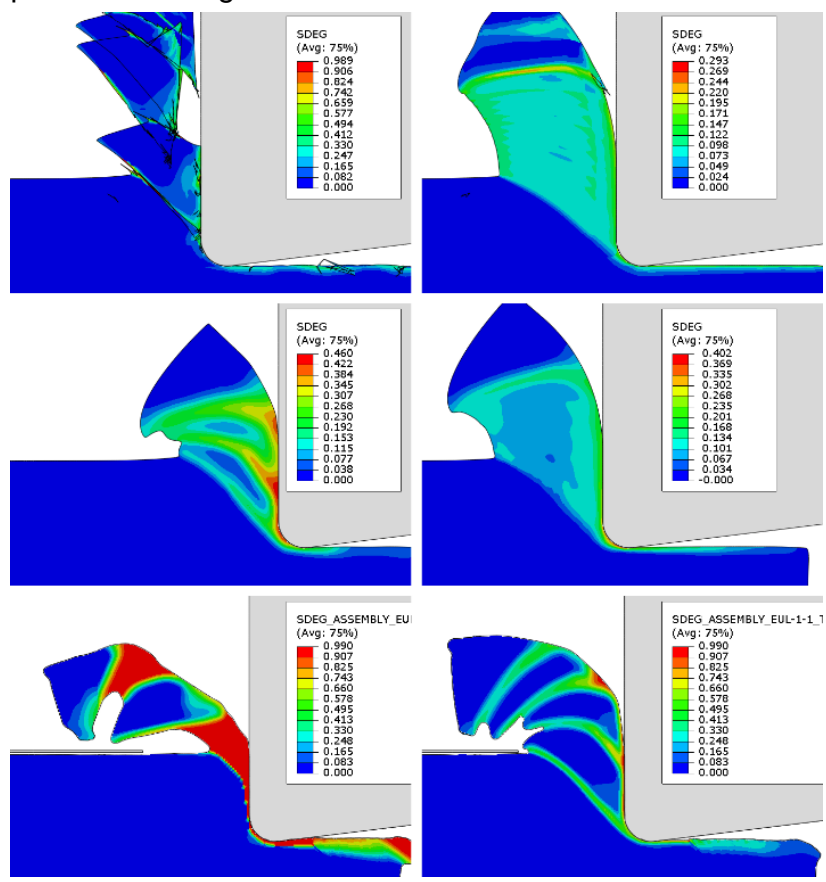

Fig. 13. Fracture energy variation: left column: $-10 \mathrm{~N} / \mathrm{mm}$; right column: $+10 \mathrm{~N} / \mathrm{mm}$. Element deletion - remeshing script - CEL. Degradation variable $D$

An increase in fracture energy leads to flow chip formation in the element deletion model and the remeshing model. The CEL-model shows a proper segmented chip, and the completely degraded material is no longer present. This shows that the value of the fracture energy must considered with the respective simulation model in mind.

\section{SUMMARY}

The usage of mesh-based simulation models to depict adiabatic shear banding has been demonstrated. The difference of the models has been shown. There is a difference in the behaviour of the models and that this behaviour must be kept in mind. The remeshing model 

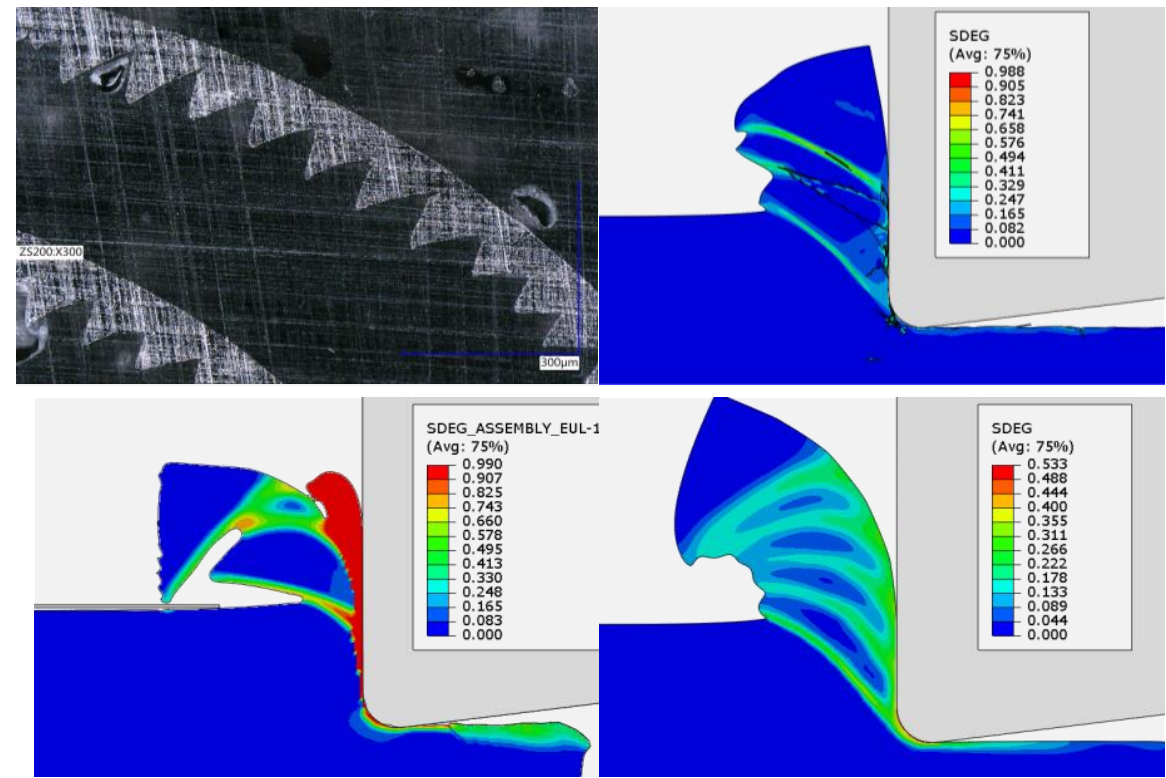

seems to yield better and clearer depictions of both the feed- and cutting force, whereas the CEL-Model seems better suited to examine the chip morphology. The improved element deletion model is a good in-between, but the negative effects of element deletion must be considered.
Future works will see the usage of an improved materialand friction model.

\section{ACKNOWLEDGMENTS}

The authors thank the DFG for this funding and intensive technical support.

Fig. 14: From upper left to down right: experiment - element deletion - CEL - remeshing

\section{REFERENCES}

[Arrazola, 2010]. Arrazola, P.J. and Özel, T., Investigations on the effects of friction modeling in finite element simulation of machining, International Journal of Mechanical Sciences 2010, Vol. 52 No. 1, pp. 31-42.

[Bäker, 2004]. Bäker, M., Finite Element Simulation of Chip Formation, Gemeinsame Fakultät für Maschinenbau und Elektrotechnik, Braunschweig, Technische Universität Carolo-Wilhelmina zu Braunschweig, Gemeinsame Fakultät für Maschinenbau und Elektrotechnik, 2004.

[Benson, 2004]. Benson, D.J. and Okazawa, S., Contact in a multi-material Eulerian finite element formulation, Computer Methods in Applied Mechanics and Engineering 2004, Vol. 193 39-41, pp. 4277-4298.

[Cervay, 1974]. Cervay, R.R., Mechanical properties of Ti$6 \mathrm{Al}-4 \mathrm{~V}$ annealed forgings.

[Ducobu, 2017]. Ducobu, F., et al., On the importance of the choice of the parameters of the Johnson-Cook constitutive model and their influence on the results of a Ti6Al4V orthogonal cutting model, International Journal of Mechanical Sciences 2017, Vol. 122, pp. 143-155.

[Heisel, 2013]. Heisel, U., et al., Modelling of orthogonal cutting processes with the method of smoothed particle hydrodynamics, Production Engineering 2013, Vol. 7 No. 6, pp. 639-645.

[Hillerborg, 1976]. Hillerborg, A., et al., Analysis of crack formation and crack growth in concrete by means of fracture mechanics and finite elements. Division of Building Materials, Lund Institute of Technology, Lund, Sweden, CEMENT and CONCRETE RESEARCH 1976, Vol. 1976 Vol. 6, pp. 773-782.
[Johnson]. Johnson, G.R. and Cook, W.H., A constitutive model and data for metals subjected to large strains, high strain rates and high temperatures.

[Jomaa, 2017]. Jomaa, W., et al., Finite element simulation and analysis of serrated chip formation during high-speed machining of AA7075-T651 alloy, Journal of Manufacturing Processes 2017, Vol. 26, pp. 446-458.

[Klocke, 2017]. Klocke, F., et al., Identification of friction and material model parameters for finite element simulation of TiAl6V4. Reibung, Schmierung und Verschleiß 2017, Reibung, Schmierung und Verschleiß 66-1-66-9.

[Liu, 2017]. Liu, R., et al., An Investigation of Side Flow during Chip Formation in Orthogonal Cutting, Procedia Manufacturing 2017, Vol. 10, pp. 568-577.

[Mabrouki, 2008]. Mabrouki, T., et al., Numerical and experimental study of dry cutting for an aeronautic aluminium alloy (A2024-T351), International Journal of Machine Tools and Manufacture 2008, Vol. 48 No. 11, pp. 1187-1197.

[Möhring, 2018]. Möhring, H.-C., et al., Temperature calculation in cutting zones, CIRP Annals 2018, Vol. 67 No. 1, pp. 61-64.

[Öpöz, 2016]. Öpöz, T.T. and Chen, X., Chip Formation Mechanism Using Finite Element Simulation, Strojniški vestnik - Journal of Mechanical Engineering 2016, Vol. 62 No. 11 , pp. $636-646$

[Rana, 2019]. Rana, P., et al., Orthogonal Turning Simulations for Casted Steel Alloy Using Mesh Free Methods 2019, pp. 337-346.

[Schulze, 2011]. Schulze, V. and Zanger, F., Numerical Analysis of the Influence of Johnson-Cook-Material Parameters on the Surface Integrity of Ti-6Al-4V, Procedia Engineering 2011, Vol. 19, pp. 306-311. 
[Soliman, 2020]. Soliman, H.A., et al., Investigation of process parameters in orthogonal cutting using finite element approaches, Heliyon 2020, Vol. 6 No. 11, e05498.

[Sridhar, 2020]. Sridhar, P., et al., Discretization approaches to model orthogonal cutting with Lagrangian, Arbitrary Lagrangian Eulerian, Particle Finite Element method and Smooth Particle Hydrodynamics formulations, Procedia CIRP 2020, Vol. 93, pp. 1496-1501.

[Umbrello, 2008]. Umbrello, D., Finite element simulation of conventional and high speed machining of Ti6Al4V alloy, Journal of Materials Processing Technology 2008, pp. 79-87.

[Zhang, 2021]. Zhang, C. and Choi, H., Study of segmented chip formation in cutting of high-strength lightweight alloys, The International Journal of Advanced Manufacturing Technology 2021, Vol. 112 9-10, pp. 26832703. 